REVISIONES

Rev Obstet Ginecol Venez. 2021; 81 (4): 390-405. https://doi.org/10.51288/00810411

\title{
Técnicas quirúrgicas en cesárea segmentaria. Revisión de evidencias
}

\author{
Domenico Guariglia. ${ }^{1}$
}

\begin{abstract}
RESUMEN
Objetivo: Establecer cuáles han sido las variaciones de la técnica de Pfannenstiel-Kerr y su influencia en la morbimortalidad materna.

Métodos: Se realizó una búsqueda electrónica en PubMed, Medline y Cochrane, de trabajos en idiomas inglés y español, sin importar el pais de origen, preferiblemente aleatorizados y controlados, realizados entre 2010 y 2020, sobre variaciones en la técnica quirúrgica original, con énfasis en las diferencias entre las técnicas Pfannenstiel-Kerr y Miglav-Ladash.

Resultados: Las variaciones más importantes, en lo relativo a la morbi-mortalidad, fueron: 1. Preferir la incisión cutánea transversa, en especial tipo Joel Cohen; 2. Disección roma lateral del subcutáneo y cráneo-caudal de la aponeurosis, posterior a su incisión; 3. Separación digital y lateral de los músculos rectos abdominales; 4. Prolongar digitalmente y lateralmente la histerotomía; 5. Histerorrafia, sin exteriorizar el útero, en uno o dos planos de sutura, indiferentemente; 6 . No suturar el peritoneo visceral y parietal ni aproximar los rectos abdominales; 7. Cierre del subcutáneo a puntos separados si el espesor en mayor de $2 \mathrm{~cm}$.

Conclusiones: Las modificaciones en la técnica original lograron una disminución estadísticamente significativa del tiempo operatorio, de la cantidad de suturas, del requerimiento de analgésicos, de la pérdida sanguínea, de la morbilidad febril, así como de la infección de herida operatoria y de la estancia hospitalaria.
\end{abstract}

Palabras clave: Cesárea, Técnica quirúrgica, Pfannenstiel-Kerr, Misgav-Ladach.

\section{SUMMARY}

Aim: To know which have been the variations of the Pfannenstiel-Kerr technique and how they have influenced maternal morbidity and mortality.

Methods: An electronic search was carried out in PubMed, Medline and Cochrane, of works in English and Spanish languages, regardless of the country of origin, preferably randomized and controlled between 2010 and 2020, on variations in the original surgical technique, with emphasis on the differences between the PfannenstielKerr and Miglav-Ladash techniques.

Results: The more importants variations regarding morbidity and mortality were: 1. Preferring the transverse skin incision, especially the Joel Cohen type; 2. Lateral blunt dissection of the subcutaneous and cranio-caudal aponeurosis, after its incision; 3. Digital and lateral separation of the rectus abdominis muscles; 4. Digitally and laterally prolong the hysterotomy; 5 . Uterine incision closure, without externalizing the uterus, in one or two suture planes, indifferently; 6. Do not suture the visceral and parietal peritoneum or approach the rectus abdominis; 7. Closure of the subcutaneous to separate points, if the thickness is greater than $2 \mathrm{~cm}$.

Conclusions: Modifications to the original technique achieved a statistically significant decrease in operative time, the number of sutures, the requirement for analgesics, blood loss, febrile morbidity, as well as operative wound infection and hospital stay.

Keywords: Cesarean section, Surgical technique, Pfannenstiel-Kerr, Misgav-Ladach.

${ }^{1}$ Profesor Titular Cátedra de Obstetricia B. Escuela de Medicina Luis Razetti. Universidad Central de Venezuela. Correo de correspondencia: dguarigliag@yahoo.com

Forma de citar este artículo: Guariglia D. Técnicas quirúrgicas en cesárea segmentaria. Revisión de evidencias. Rev Obstet Ginecol Venez. 2021; 81(4):390-405. https://doi.org/10.51288/00810411

\section{INTRODUCCIÓN}

La operación cesárea es la intervención quirúrgica obstétrica más frecuente en la mayoría de los países, a 


\section{TÉCNICAS QUIRÚRGICAS EN CESÁREA SEGMENTARIA. REVISIÓN DE EVIDENCIAS}

pesar de que su morbimortalidad es mayor que la del parto normal. La técnica quirúrgica más utilizada, conocida como Pfannenstiel-Kerr $(1,2)$ por el tipo de incisión de la laparotomía y por la incisión a nivel del segmento uterino, se ha mantenido durante muchas décadas; sin embargo, en los últimos 20 años ha habido una revisión del procedimiento, tratando de simplificar la técnica antes mencionada. De ahí que, a partir de la propuesta de Stark (3), conocida como técnica de Misgav-Ladach y presentada en el Congreso de la Federación Internacional de Ginecología y Obstetricia (FIGO) en 1994 (4), utilizando la incisión abdominal de Joel-Cohen (5), se han realizado una gran cantidad de ensayos clínicos, muchos de ellos aleatorizados y controlados, a fin de probar las distintas variantes técnicas de la intervención y su impacto sobre el riesgo de complicaciones maternas a corto, mediano y largo plazo.

Debido a la alta frecuencia de esta intervención y a la multiplicidad de ensayos clínicos en las últimas dos décadas, donde se describen las diferentes variaciones de la técnica original, el autor ha considerado que, con sustentación en la medicina basada en la evidencia, sería importante conocer cuáles han sido estas variantes y en qué han influido, si es el caso, en la disminución de la morbimortalidad materna.

En este trabajo, se realizó una búsqueda electrónica en las bases de datos de PubMed, Medline y revisiones Cochrane, de los trabajos publicados sobre técnicas quirúrgicas en la cesárea, entre 2010 y 2020, preferiblemente aleatorizados y controlados; sus ventajas y desventajas, con especial énfasis en las diferencias entre las dos técnicas actuales: Pfannenstiel-Kerr y Misgav-Ladash. Las búsquedas electrónicas se restringieron a los idiomas inglés y español, sin importar el país de origen de los estudios.

Vol. 81, $\mathrm{N}^{\mathrm{0}}$ 4, diciembre 2021.

\section{Tipos de cesáreas}

Hay fundamentalmente 2 tipos de cesáreas: la intraperitoneal y la extraperitoneal. La cesárea extraperitoneal, realizada antes de la era antibiótica, con abordaje del segmento uterino por vía abdominal o vaginal, mediante disección paravesical o supravesical, ha sido abandonada, por las graves complicaciones como hemorragia, lesión de la vejiga o la posterior formación de fístulas vésicovaginales $(6,7)$. Actualmente, totalmente en desuso, ha habido intenciones de actualizarla en ensayos clínicos no randomizados, sobre todo en la extracción de feto muerto o no viable $(8,9)$.

Las técnicas intraperitoneales se clasifican, de acuerdo al sitio de la incisión en el útero en: a) Segmentaria, la cual puede ser transversa o vertical y b) Corporal. Actualmente la más utilizada en más del $98 \%$ de los casos, es la cesárea segmentaria transversa tipo Kerr (2), con incisión de piel transversal tipo Pfannenstiel (1).

La técnica clásica de Pfannenstiel-Kerr consiste en:

1. Incisión de la piel a 2,5-3 cm por encima de la sínfisis del pubis, transversal baja con ligera curva hacia arriba, de 10 a $15 \mathrm{~cm}$.

2. Incisión del tejido celular subcutáneo con bisturí o electrobisturí.

3. Se hace una incisión hacia uno y otro lado de la línea media de la vaina de los rectos, la cual se extiende hacia los lados con tijera curva de mayo. Los bordes superiores e inferiores de la aponeurosis se toman con pinzas de Kocher y se separa la vaina de los músculos rectos, mediante disección roma y cortante. Se suturan o electrocoagulan los vasos perforantes.

4. Se separan los músculos rectos en la línea media 
mediante disección digital o cortante, si están adheridos.

5. El peritoneo parietal se toma con 2 pinzas de Crile en su parte más alta, a fin de evitar lesionar la vejiga y cuidándose de la presencia de asas intestinales. Se secciona con tijera verticalmente de arriba hacia abajo.

6. Incisión del peritoneo visceral, rechazando el fondo vesical.

7. La incisión del miometrio se realiza en el segmento uterino, en forma transversal rectilínea, de aproximadamente $2 \mathrm{~cm}$ con bisturí; luego, introduciendo uno o dos dedos de ambas manos en la incisión, se separan las fibras musculares transversalmente, o se prolonga la incisión en la misma dirección con tijera, arqueando un poco en los extremos, para evitar lesiones de la arteria uterina o sus ramas.

8. Extracción del feto, alumbramiento y revisión de la cavidad uterina.

9. Histerorrafia en un solo plano, continua o a puntos separados o, más frecuentemente, continua en 2 planos, uno perforante y otro invaginante.

10. Se sutura el peritoneo visceral con sutura continua catgut crómico 00 .

11. Limpieza de la cavidad pelviana y revisión de ovarios y trompas.

12. Sutura del peritoneo parietal con sutura continua con catgut crómico 00 .

13. Afrontamiento de músculos rectos a puntos separados con catgut crómico 00.

14. Cierre del plano aponeurótico, con sutura continua, 0 o 1 , poliglactina.
15. Cierre del plano subcutáneo, con puntos separados, catgut simple 00 .

16. Cierre de piel con sutura intradérmica continua o a puntos separados, con sutura no absorbible.

Michael Stark describe en 1994 (3) una técnica simplificada de cesárea, conocida con el nombre del hospital israelí donde se realizó: Misgav-Ladach. En esta técnica, se rechaza el uso de instrumentos afilados y se prefiere la manipulación manual. Las modificaciones más importantes, con respecto a la técnica de Pfannenstiel-Kerr, han sido originalmente $(3,4)$ :

1. La incisión cutánea transversal recta (incisión de Joel Cohen): un poco más alta que la de Pfannenstiel (4-5 cms por encima de la sínfisis púbica).

2. La incisión del tejido celular subcutáneo se realiza en su centro con el bisturí hasta llegar a la superficie de la aponeurosis, para luego separar digital y lateralmente la grasa subcutánea. Esto se asocia a menor tiempo operatorio y menor posibilidad de dañar vasos en los extremos de la herida y/o filetes nerviosos.

3. La apertura de la aponeurosis, previa incisión transversa central de 3 a $5 \mathrm{~cm}$, o de 2 pequeñas incisiones simétricas, se realiza mediante la introducción de 2 dedos, que divulsionan la fascia primero hacia los extremos y luego en dirección céfalo-caudal, a fin de lograr su separación completa de los músculos rectos abdominales.

4. El músculo recto abdominal se separa digitalmente y en dirección lateral. Esto se logra porque, con la incisión de Cohen, no hay necesidad de disecar los músculos piramidales ni disección amplia del músculo recto, como en la incisión de Pfannenstiel. 


\section{TÉCNICAS QUIRÚRGICAS EN CESÁREA SEGMENTARIA. REVISIÓN DE EVIDENCIAS}

5. El peritoneo se abre, en la parte más alta, estirándolo con ambos dedos índices o con la ayuda de una pinza de Crile, para luego abrirlo digital y verticalmente.

6. El útero, previa incisión central directa en el segmento, se abre con el dedo índice de una mano y el pulgar de la otra, en sentido lateral. No se disecciona el peritoneo visceral.

7. Extracción fetal.

8. Extracción de la placenta por tracción continua y masaje uterino fúndico. Originalmente el método proponía extracción manual de la placenta.

9. Revisión de la cavidad uterina con gasas.

10. El útero se cierra en un solo plano con una sola sutura continua.

11. El peritoneo visceral y parietal no se cierran.

12. Limpieza de la cavidad pelviana y revisión de ovarios y trompas.

13. El músculo recto abdominal no se aproxima con sutura.

14. La fascia del recto se cierra con una sutura continua poliglactina 1.

15. El tejido celular subcutáneo, se cierra con puntos separados, si su espesor es mayor de $2 \mathrm{~cm}$, con catgut simple 00 .

16. La piel se cierra con 2 o 3 sutura a puntos separados.

A partir de ese año han aparecido publicadas múltiples revisiones en varios países de la técnica de Misgav-Ladach comparada con la técnica habitual de Pfannnenstiel- Kerr, así como otras variaciones sobre aspectos generales y particulares de la técnica original. De la base de datos Cochrane de revisiones sistemáticas, se escogieron 6 revisiones, todas de trabajos aleatorizados y controlados de los años 2012, 2013, 2014 y 2020 y de PubMed/ Medline se escogieron 4 trabajos: 1 aleatorizado de 2011, 1 prospectivo de cohorte de 2012, y 1 aleatorizado y controlado, multicéntrico de 6 países iniciado en 2013 y finalizado en 2016, conocido como Coronis Trial (Cuadros 1 - 5).

Técnica de Misgav-Ladash modificada por Stark versus técnica de Pfannenstiel-Kerr

De la revisión de los trabajos aleatorizados y controlados publicados en el lapso antes mencionado, en los cuales se compara esta nueva técnica simplificada con la técnica más comúnmente realizada que es la de Pfannenstiel-Kerr, en dos estudios aleatorizados se encontró que, con la técnica de Misgav-Ladash se acorta significativamente el tiempo operatorio, hay menor uso de suturas y menor dolor posoperatorio $(10,11)$ (Cuadro 1). Sin embargo, el estudio más importante fue iniciado en 2013 (12) y finalizado con un periodo de seguimiento de 3 años, en 2016 (13). En este estudio internacional, multicéntrico, aleatorizado y controlado donde participaron 6 países, que incluyó 13153 pacientes, tras 3 años de seguimiento, se compararon varios parámetros entre las 2 técnicas quirúrgicas, como: la disección roma versus cortante de tejido celular subcutáneo y de la aponeurosis; la exteriorización del útero versus útero intrabdominal para la realización de la histerorrafia; la histerorrafia en una capa versus 2 capas; el cierre o no del peritoneo visceral y parietal. Los autores no encontraron diferencias significativas en estos parámetros, pero si encontraron una ventaja estadísticamente significativa, con la técnica de Misgav-Ladach, en lo relativo a menor tiempo quirúrgico, menor pérdida hemática, menor dolor posoperatorio y menor incidencia de fiebre puerperal (Cuadro 1). 
Cuadro 1. PUBMED/ MEDLINE

Técnica quirúrgica en cesárea

\begin{tabular}{|c|c|c|c|c|}
\hline Año & Autor & Tipo y n de ensayos & $\mathrm{N}$ de casos & Parámetro evaluado \\
\hline 2011 & Naki y cols. (10) & A & 180 & $\begin{array}{c}\text { Misgav-Ladash vs Pfannenstiel-Kerr } \\
\boldsymbol{\nabla} \text { Tiempo operatorio } \mathrm{p}<0,001 \\
\boldsymbol{\nabla} \text { Material de sutura } \mathrm{p}<0,001\end{array}$ \\
\hline 2012 & Hudic y cols. (11) & Observacional/ Cohorte & 4944 & $\begin{array}{c}\text { Misgav-Ladash vs Pfannenstiel-Kerr } \\
\boldsymbol{\nabla} \text { Tiempo operatorio } \mathrm{p}<0,005 \\
\boldsymbol{\nabla} \text { Material quirúrgico } \mathrm{p}<0,005 \\
\boldsymbol{\nabla} \text { Analgésicos } \mathrm{p}<0,005 \\
\boldsymbol{\nabla} \text { Morbilidad febril } \mathrm{p}<0,005 \\
\boldsymbol{\nabla} \text { Infección herida } \mathrm{p}<0,005\end{array}$ \\
\hline \multirow{5}{*}{$\begin{array}{l}2013 \\
2016\end{array}$} & \multirow{5}{*}{$\begin{array}{l}\text { Abalos y cols. (12) } \\
\text { Ábalos y cols. (13) }\end{array}$} & \multirow{5}{*}{ A/C CORONIS TRIAL } & \multirow{5}{*}{15935} & $\begin{array}{l}\text { Histerotomía digital vs cortante } \\
\mathrm{RR}=1,03 \text { (CI } 0,91-1,1795 \% \mathrm{NS}\end{array}$ \\
\hline & & & & $\begin{array}{c}\text { Histerorrafia intra vs extraabdominal } \\
\quad \mathrm{RR}=0,96 \text { (IC } 0,84-1,0895 \%) \mathrm{NS}\end{array}$ \\
\hline & & & & $\begin{array}{l}\text { Sutura en una capa o dos capas } \\
R R=0,96(\text { IC } 0,85-1,0895 \%) \mathrm{NS}\end{array}$ \\
\hline & & & & $\begin{array}{l}\text { No sutura vs sutura de peritoneo NS } \\
\qquad R R=1,06 \text { (IC } 0,94-1,2095 \% \text { ) }\end{array}$ \\
\hline & & & & $\begin{array}{c}\text { Sutura con catgut vs poliglactina NS } \\
\quad \mathrm{RR}=0,90 \text { (IC } 0,78-1,0495 \%)\end{array}$ \\
\hline
\end{tabular}

$\mathrm{A}=$ Aleatorizado; $\mathrm{A} / \mathrm{C}=$ Aleatorizado y controlado; $\mathrm{RR}=$ Riesgo relativo; $\mathrm{IC}=$ Intervalo de confianza; $\boldsymbol{\nabla}=\mathrm{Disminución}$; NS= No estadísticamente significativo

A continuación, se pasará revista detallada a los pasos quirúrgicos más importantes en la realización de la cesárea intraperitoneal segmentaria transversa, haciendo notar algunas de las diferencias entre ambas técnicas, anteriormente mencionadas, sus ventajas y desventajas, así como las nuevas evidencias surgidas en los últimos años sobre la técnica quirúrgica de la cesárea en general.

\section{Preparación del campo quirúrgico y piel}

Con relación a la preparación y medidas antisépticas de la piel y el campo quirúrgico, previas a la cesárea; en un ensayo clínico de 2016, de Tuuli y cols. (14) sobre 1147 pacientes, se concluyó que la clorhexidina alcohólica era significativamente mejor, en cuanto a la prevención de infección de la herida quirúrgica, que el preparado povidona-alcohol (14). En una revisión Cochrane de 2020, actualización de una similar de 2014, se hicieron comparaciones en 13 ensayos aleatorizados controlados entre diferentes agentes antisépticos (p.ej., alcohol, povidona yodada); diferentes métodos de aplicación de los antisépticos (p.ej., lavado, tinturas, paños); diferentes formas de antisépticos (p.ej., polvo, líquido); y también entre diferentes paquetes de preparación de la piel incluyendo una mezcla de agentes y métodos: como un paño plástico para la incisión, que puede o no estar impregnado con agentes antisépticos. Una evidencia moderada indicó que el gluconato de clorhexidina, comparado con la povidona yodada, disminuye ligeramente la incidencia de la infección 


\section{TÉCNICAS QUIRÚRGICAS EN CESÁREA SEGMENTARIA. REVISIÓN DE EVIDENCIAS}

Cuadro 2. Base de datos Cochrane de revisiones sistemáticas

Técnica quirúrgica en cesárea. Preparación del campo quirúrgico. Tipo de incisión.

\begin{tabular}{|c|c|c|c|c|}
\hline Año & Autores & Tipo y n de ensayos & $\mathrm{N}$ casos & Parámetros y resultados \\
\hline & & & & $\begin{array}{l}\text { Clorhexidine gluconato vs iodopovidona } \\
\text { (Preparación de la piel) }\end{array}$ \\
\hline 2020 & Hadiati y cols. (15) & $\mathrm{A} / \mathrm{C}$ & 4.323 & $\begin{array}{c}\nabla \text { Infección herida operatoria } \\
\text { RR 0,72 (IC 0,58-0,91 95\%) } \\
\text { Endometritis } \\
0.95 \text { ( IC } 0,49-1,8695 \% \text { ) NS }\end{array}$ \\
\hline 2013 & Mathai y cols. (17) & $\mathrm{A} / \mathrm{C} 2$ & 411 & 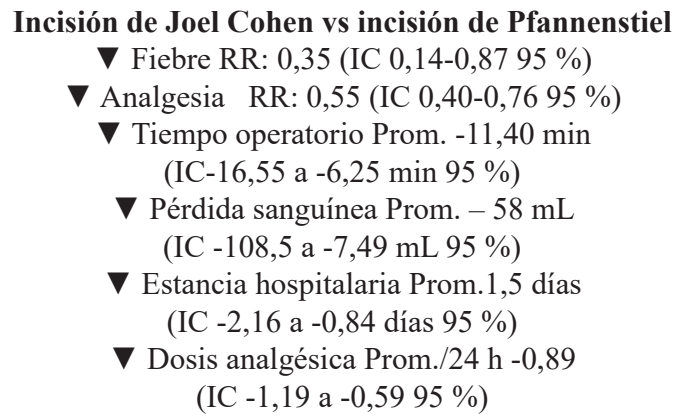 \\
\hline
\end{tabular}

$\mathrm{A}=$ Aleatorizado; $\mathrm{A} / \mathrm{C}=$ Aleatorizado y controlado; $\mathrm{RR}=$ Riesgo relativo; $\mathrm{IC}=$ Intervalo de confianza; $\boldsymbol{\nabla}=$ Disminución;

$\boldsymbol{\Delta}=$ Aumento. $\mathrm{N}=$ ddisminución $\mathrm{S}=$ No estadísticamente significativo

del sitio quirúrgico (RR 0,72; IC del $95 \%$ : 0,58 a $0,91)$. Se concluyó, sin embargo, que no existe evidencia clara sobre cuál es la preparación cutánea más eficaz en cuanto a prevención de la infección posquirúrgica en la cesárea (15) (Cuadro 2).

2. Incisión de la piel y apertura de la pared abdominal

Antes, la incisión media infraumbilical era la más utilizada; a finales del siglo XIX y principio del siglo $\mathrm{XX}$ se empezaron a usar las incisiones transversales. El paso más importante lo dio Pfannenstiel, citado por Ventura (1), cuando presentó su técnica, cuya mayor ventaja era la disminución de las hernias incisionales o eventraciones y mejor aspecto estético $(1,16)$. Hoy, raramente se usa la incisión media infraumbilical, siendo su ventaja más importante el mejor acceso al campo quirúrgico y la posibilidad de su extensión hacia arriba, cuando hay complicaciones. A pesar del mejor aspecto estético y menor riesgo de hernias incisionales, las incisiones transversales se vinculan con un mayor riesgo de hematomas supraaponeuróticos, por el corte de capilares perforantes, así como de lesión de nervios sensoriales cutáneos que llevan a alteraciones sensitivas en la zona (Cuadros 2 y 3). Existen otras incisiones cutáneas abdominales transversales, además de la de Pfannenstiel (7):

A) Pfannenstiel. Incisión transversal baja apenas curvilínea de 10-12 cm. A nivel de la línea media de implantación del vello púbico y se extiende un poco más allá de los bordes laterales de los músculos rectos.

B) Maylard: Incisión transversal similar a la Pfannenstiel, pero se seccionan las vainas y los músculos rectos abdominales. Permite un acceso más amplio del abdomen, pero provoca mayor daño tisular. 
Cuadro 3. Cuadro comparativo entre las incisiones cutáneas media infraumbilical, Pfannenstiel y Joel Cohen

\begin{tabular}{lccc}
\hline Tipo & Media infraumbilical & Pfannenstiel & Joel cohen \\
\hline Frecuencia de dehiscencia & Mayor & Menor & Menor \\
Frecuencia de hernia incisional & Mayor & Menor & Menor \\
Acceso al abdomen & Mejor & Peor & Peor \\
Prolongación & Si & No & No \\
Frecuencia de hematoma supraaponeurotico & Menor & Mayor & Menor \\
Frecuencia de alteraciones sensitivas locales & Menor & Mayor & Menor \\
Aspecto estético & Peor & Mejor & Mejor \\
Dolor & Menor & Mayor & Menor \\
\hline
\end{tabular}

C) Joel Cohen: Incisión transversal rectilínea a 3 a 5 cms por encima del pubis, utilizada en la técnica de Misgav-Ladach (7).

D) Cherney. Mucho más cruenta que las anteriores, es una incisión para grandes operaciones abdominales. La incisión cóncava se inicia un poco por debajo de las espinas antero superiores de la pelvis, pasando cerca del pubis. Contempla la sección de los músculos piramidales y la sección y desinserción del tendón de los rectos abdominales en el pubis.

En una revisión de Cochrane de 2013, para determinar los efectos beneficiosos y los riesgos de los métodos alternativos de incisiones quirúrgicas abdominales para la cesárea, se incluyeron 4 estudios, para un total de 411 pacientes, donde se obtuvo que, con la incisión de Joel Cohen, había una reducción de necesidades de analgésicos posoperatorios, del tiempo quirúrgico, del tiempo para la extracción fetal, de la dosis total de analgésicos en las primeras 24 horas, de la pérdida de sangre y de la estancia hospitalaria posoperatoria materna, en comparación con el grupo con la incisión de Pfannenstiel (17). En la misma revisión, con 97 pacientes, comparando la técnica de incisión de Maylard (corte del músculo recto abdominal) con la incisión de Pfannenstiel, no se informaron diferencias en la morbilidad febril, la necesidad de transfusión, la infección de la herida y las pruebas de fuerza muscular abdominal a los 3 meses posteriores a la estancia hospitalaria (17) (Cuadro 2).

En una revisión sistemática de la literatura Gizzo y cols. (18), comparando las diversas incisiones transversales, encontraron que la incisión de Joel Cohen de la técnica de Misgav-Ladach estaba asociada a una reducción del dolor agudo y crónico poscesárea. Belci y cols. (19), en el seguimiento de pacientes 5 años después de operadas por cesárea, según diferentes técnicas de incisiones transversales, encontraron igualmente que con la incisión de Joel Cohen los resultados fueron estadísticamente mejores en lo relativo a una menor intensidad del dolor, agudo o crónico y el neuropático.

\section{¿Bisturí o electrobisturí?}

El bisturí es el instrumento tradicionalmente usado en la incisión de la piel, sin embargo, se ha probado el uso de electrobisturí para este tiempo quirúrgico. En un estudio prospectivo aleatorizado comparativo del bisturí vs el electrobisturí, de 200 casos de cesáreas, observaron cambios estadísticamente significativos, con el uso del electrobisturí: menor sangrado de la herida, menor tiempo de ejecución de la incisión y menor dolor posoperatorio. No hubo diferencias en el cierre de la herida ni complicaciones infecciosas (20). Igual resultado se obtuvo en un metaanálisis en cirugía de hernias inguinales, comparando los dos métodos 


\section{TÉCNICAS QUIRÚRGICAS EN CESÁREA SEGMENTARIA. REVISIÓN DE EVIDENCIAS}

(21). A pesar de los resultados anteriores, la escogencia sobre el uso de uno de los métodos, depende mucho de la experiencia y preferencia del cirujano.

Apertura digital versus cortante del tejido celular subcutáneo

En la técnica de Misgav-Ladash, la apertura del tejido subcutáneo se asocia a menor tiempo operatorio y menor posibilidad de dañar vasos o filetes nerviosos $(3,4)$.

\section{Apertura digital versus cortante de la aponeurosis}

En la técnica de Pfannenstiel-Kerr, la apertura de la aponeurosis se realiza mediante una incisión transversal central, la cual se prolonga hacia los lados con tijera o electrobisturí. En la técnica MisgavLadach, la fascia, previa incisión transversa central, la apertura se hace mediante divulsión lateral y céfalocaudal. En un trabajo aleatorizado y doble ciego, donde se comparó la apertura cortante con tijera de la aponeurosis vs la apertura con tracción digital, se encontró que en el grupo de pacientes en los que realizó la tracción digital, el dolor crónico a largo plazo y el dolor neuropático, eran significativamente menores (22). Se supone que la extensión digital de la aponeurosis evita la lesión de vasos y de nervios superficiales de la zona.

Disección digital versus cortante de músculo recto abdominal

La disección roma y separación digital en sentido lateral de los músculos rectos abdominales, sin comprometer a los músculos piramidales, sobre todo en pacientes en su primera cesárea, a fin de exponer el peritoneo parietal, en la técnica de Misgav-Ladach, evita una mayor pérdida sanguínea, dolor posoperatorio y ahorra tiempo quirúrgico (23).

\section{Apertura del peritoneo parietal}

En la técnica simplificada, el peritoneo parietal se trata de abrir digitalmente empezando en la parte más alta del mismo, evitando el corte con tijera o electro bisturí. Se supone que con esta técnica hay menor riesgo de lesión intestinal y de vejiga. Más adelante, se analizarán las ventajas o desventajas de la apertura o cierre de ambos peritoneos

\section{Histerotomía}

Una vez en la cavidad abdominal, en la técnica de Misgav-Ladach, no recomiendan la disección del peritoneo visceral ni disecar el fondo vesical o plica vesical; esto último trae como ventaja un menor tiempo quirúrgico, menor riesgo de infección, reduce la pérdida hemática y la necesidad de analgésicos posoperatorios, según una revisión de la literatura de Mahajan (24). La separación de la plica vesical o fondo vesical se debe realizar cuando se espera una intervención laboriosa por feto macrosómico, ante la presencia de varias cesáreas anteriores, placenta previa anterior, miomas a nivel del segmento, que pudieran conllevar a lesión vesical $(6,7)$. Tampoco se recomienda la disección de la porción inferior del músculo recto anterior del abdomen y de los músculos piramidales, ya que está asociada a un mayor dolor y sangrado posoperatorio (25).

Apertura cortante versus apertura digital del segmento uterino

La incisión uterina más frecuentemente utilizada es la transversa baja a nivel del segmento uterino, que tiene como ventajas una menor pérdida sanguínea y un menor riesgo de ruptura en embarazos posteriores $(6,7)$. Existen también incisiones verticales del útero, como son: la incisión vertical baja, a nivel del segmento uterino y la incisión vertical clásica, que se 
realiza a nivel del cuerpo uterino o fundus, ambas muy poco usadas actualmente $(6,7)$.

En un estudio comparativo de 2 grupos de pacientes: grupo A con 136 casos, a quienes se les realizó la histerotomía a nivel del segmento uterino mediante apertura digital, previa pequeña incisión con bisturí, y grupo B, con 78 casos, donde la extensión de la incisión se realizó mediante tijera curva, se encontró que, con esta última técnica, había un mayor descenso de la hemoglobina, así como una mayor tendencia a laceraciones miometriales y lesión de la arteria uterina por extensión (26). Igual resultado, estadísticamente significativo, se obtuvo en un estudio prospectivo aleatorizado, que incluyó 1076 casos (27).

En una revisión de Cochrane de 2014, que incluyó 5 estudios, con un total de 2141 pacientes, donde se comparó la disección roma versus cortante al realizar la incisión uterina, no se encontró una diferencia estadísticamente significativa con relación a la morbilidad febril, pero la pérdida sanguínea media y la necesidad de transfusión fueron significativamente menores luego de la extensión roma de la incisión (28) (Cuadro 4).

No se ha encontrado ningún beneficio en la permeabilización digital del orificio cervical interno del cuello uterino, posterior al alumbramiento y antes de la histerorrafia (29).

\section{Histerorrafia}

Sutura a puntos separados versus continua. Un solo plano versus dos planos. Sutura con exteriorización del útero versus útero intraabdominal.

Cuadro 4. Base de datos Cochrane de revisiones sistemáticas Técnica quirúrgica en cesárea. Histerotomía

\begin{tabular}{|c|c|c|c|c|}
\hline Año & Autores & Tipo y $\mathrm{n}$ de ensayos & $\mathrm{N}$ casos & Parámetros y resultados \\
\hline \multirow{13}{*}{2014} & \multirow{13}{*}{ Dodd y cols. (28) } & & & Histerotomía: incisión roma vs cortante \\
\hline & & $\mathrm{A} / \mathrm{C} \quad 4$ & 1941 & Morbilidad febril: RR=0,86 (IC 0,70- 1,05 95\%) NS \\
\hline & & $\mathrm{A} / \mathrm{C} 2$ & 1145 & $\boldsymbol{\nabla}$ Hemoglobina: RR= Prom - 55 (IC- 79,4 a -30,5 95\%) \\
\hline & & $\mathrm{A} / \mathrm{C} 2$ & 1.345 & $\boldsymbol{\Delta}$ Transfusión sanguínea: RR 0,24 (IC 0,09-0,62 95\%) \\
\hline & & $\mathrm{A} / \mathrm{C} \quad 1$ & 200 & Tiempo operatorio Prom - 2,80 (IC -5,84 a -0,24 95\%) NS \\
\hline & & & & $\begin{array}{c}\text { Histerotomía: extensión de la incisión: } \\
\text { transversa vs céfalo-caudal }\end{array}$ \\
\hline & & $\mathrm{A} / \mathrm{C}$ & 811 & $\Delta$ Pérdida sanguínea Prom. 42 mL (IC 1,31-82,6 95\%) \\
\hline & & & & Histerorrafia: en 1 capa vs 2 capas \\
\hline & & $\mathrm{A} / \mathrm{C}$ & 13.890 & Morbilidad febril NS \\
\hline & & $\mathrm{A} / \mathrm{C} \quad 4$ & 13.571 & Riesgo de transfusión RR 0,86 (IC 0,63- 1,17 95\%) NS \\
\hline & & & & Sutura. Catgut crómico vs Poliglactina 910 \\
\hline & & $\mathrm{A} / \mathrm{C} \quad 1(\mathrm{C})$ & 9.544 & $\begin{array}{c}\boldsymbol{\nabla} \text { Transfusión sanguínea } \\
\text { RR 0,49 (IC 0,32-0,76 95\%). } \\
\text { ₹ Complicaciones o reintervenciones } \\
\text { RR 0,58 (IC 0,37- 0,89 95\%) } \\
\end{array}$ \\
\hline & & $\mathrm{A} / \mathrm{C}$ & 300 & RR $0,92(0,38-2,2095 \%) \mathbf{N S}$ \\
\hline
\end{tabular}

$\mathrm{A}=$ Aleatorizado; $\mathrm{A} / \mathrm{C}=$ Aleatorizado y controlado; $\mathrm{RR}=$ Riesgo relativo; Prom= Promedio; $\mathrm{NS}=$ No significativo;

$\boldsymbol{\nabla}=$ Disminución; $\quad \boldsymbol{\Delta}=$ Aumento. 


\section{TÉCNICAS QUIRÚRGICAS EN CESÁREA SEGMENTARIA. REVISIÓN DE EVIDENCIAS}

En la técnica habitual de la histerorrafia, la sutura del músculo uterino puede ser a puntos separados o continuos. La sutura continua puede ser en un solo plano o en dos planos, siendo la segunda invaginante, con material catgut crómico o ácido poliglicólico. Esta última es generalmente la más usada. Siempre ha existido la controversia sobre las ventajas y desventajas de cada una de las suturas. La medida del grosor de la cicatriz del segmento uterino inferior con el ultrasonido ha sido utilizada para evaluar la calidad de la misma posterior a una cesárea y su asociación con el riesgo de ruptura uterina.

En un metaanálisis de Cochrane de 2014, que incluyó 14 estudios, comparando el cierre del útero en una sola capa versus 2 capas, no se identificaron diferencias estadísticamente significativas en la morbilidad febril $\mathrm{y}$, aunque el cierre en una sola capa se asoció con una reducción de la pérdida sanguínea media, advierten sobre la poca aplicabilidad del estudio en la clínica, por la alta heterogeneidad de los estudios (28) (Cuadro 4).

En un metaanálisis de 2017, en el que incluyen 9 ensayos clínicos (3690 pacientes), se concluye que no existen diferencias entre el riesgo de dehiscencia de la cicatriz: $0,4 \%$ en cierre en una capa vs. $0,2 \%$ doble capa con $\mathrm{RR}=1,34$ (IC 0,24-4,82 $95 \%$ ) o de rotura uterina: $0,1 \%$ en una capa y $0,1 \%$ en doble capa con $\mathrm{RR}=0,52$ (IC 0,05-5,53 95\%); aunque en las pacientes con cierre en doble capa el miometrio en la cicatriz era significativamente más grueso (30).

Vachon-Marceau y cols. (31), de un total de 1613 pacientes con antecedentes de una cesárea, repartidos en 1118 casos con doble plano de sutura y 495 con un solo plano de sutura, estimaron el impacto del tipo de sutura previa uterina con relación al espesor del segmento uterino, a fin de evaluar la calidad de la cicatriz y su asociación con el riesgo de ruptura uterina. Entre las semanas 34 y 38, se comparó el espesor del segmento uterino, determinado mediante ecografía transabdominal y transvaginal con respecto al número de planos de sutura y al tipo de sutura utilizados en los reportes de la primera cesárea. Los autores concluyeron que, comparado con el cierre en un solo plano, el cierre en dos planos, en una cesárea anterior, estaba asociado con un mayor espesor del segmento uterino en el tercer trimestre y un menor riesgo de un grosor del segmento menor de $2 \mathrm{~mm}$ en el siguiente embarazo. El tipo de sutura no tuvo impacto significativo en el espesor del segmento.

Originalmente, en la técnica de Misgav-Ladash se exterioriza el útero para la histerorrafia (3). En un metaanálisis de estudios aleatorizados y controlados de 2009, no se encontró diferencias estadísticamente significativas en la reparación intraabdominal versus la extraabdominal (32). Coutinho y cols. (33), sin embargo, observaron una diferencia estadísticamente significativa en la histerorrafia extraabdominal en lo que respecta a un menor número de suturas y un menor tiempo quirúrgico, ventajas que se vieron minimizadas por la presencia de dolor moderado a severo a las 6 horas posteriores.

\section{Cierre o no del peritoneo visceral y parietal}

Kerr preconizó, en 1926, la cesárea segmentaria y como parte de la técnica incluía la disección del peritoneo vesical y cierre posterior de ambos peritoneos, visceral y parietal (2). En las últimas dos o tres décadas se ha cuestionado si el cierre de esta parte anatómica, tanto visceral como parietal, es conveniente e indispensable. Se ha indicado que es más probable que se formen adherencias peritoneales cuando se sutura el peritoneo, posiblemente como resultado de una reacción tisular al material de la sutura. Esta discusión se ha dado tanto en cirugía ginecobstétrica como en cirugía general. Terán y col. (34), en un estudio prospectivo, descriptivo, controlado, de 5 años de duración, con 200 pacientes a quienes no se les suturaron ambos peritoneos durante 
la cesárea, comparadas con 100 pacientes a quienes sí se les realizó la síntesis, concluyeron que no cerrar el peritoneo visceraly parietal no afectaba el posoperatorio inmediato y mediato y que solo faltaba por investigar la aparición de adherencias intraabdominales en el futuro. Igual resultado obtuvieron Pagés y cols. (35) al practicar la técnica simplificada, que consistía en no suturar ambos peritoneos y el recto abdominal. MalekMellouli y cols. (36) encontraron que no cerrar ambos peritoneos acorta el tiempo operatorio, disminuye el dolor posoperatorio y favorece la recuperación más precoz del tránsito intestinal.

En una revisión Cochrane, de 2014, de 21 ensayos (17 276 pacientes), sobre los efectos del cierre o no de ambos peritoneos en los resultados intraoperatorios, posoperatorios inmediatos y a largo plazo, hubo una reducción del tiempo operatorio en todos los subgrupos, una reducción del periodo de hospitalización posterior a la cesárea, excepto en el subgrupo en el que solo se suturó el peritoneo parietal; sin embargo concluyeron que necesitan pruebas más consistentes sobre el dolor a largo plazo, la formación de adherencias y la infertilidad (37) (Cuadro 5). No se ha demostrado a largo plazo un aumento de cuadros adherenciales en cesáreas practicadas posteriormente (38). Igualmente, es beneficioso en pacientes con desórdenes hipertensivos, disfunción renal y anormalidades y disfunción del sistema autonómico, debido a la sobreactividad del sistema simpático, como consecuencia del aumento del dolor posoperatorio (39).

6. Cierre de pared abdominal

Cierre o no del peritoneo parietal y cuadros adherenciales

Se mencionaron anteriormente las ventajas y beneficios de no proceder al cierre del peritoneo visceral y parietal, sin embargo, un estudio de cohorte, realizado en 2005, concluyó que el cierre del peritoneo parietal en una paciente sometida a una primera cesárea está asociado con una significativa menor proporción de complicaciones de adherencias densas, por lo tanto, replantea revisar la conducta de no cerrar el peritoneo parietal (40).

Por otra parte, Tulandi y cols. (41), en 1988, no encontraron diferencias en lo que respecta a adherencias intraabdominales en ambos grupos (cierre del peritoneo parietal versus no cierre) cuando fueron evaluadas con una segunda laparoscopia (41).

Sutura de aproximación o no de músculos rectos abdominales

La sutura o aproximación de los músculos rectos abdominales, en las pacientes sometidas a una primera cesárea, está asociada a un aumento estadísticamente significativo de dolor posoperatorio y de mayor requerimiento de analgésicos (42).

\section{Cierre de aponeurosis}

La aponeurosis se cierra mediante sutura continua, no cruzada, ni tensa.

Aunque no hay ensayos sobre el cierre de la aponeurosis y por lo tanto no hay una evaluación basada en la evidencia, la literatura recomienda el cierre con sutura continua, no cruzada, con sutura no absorbible o de absorción retardada y con espacio entre los puntos (43).

\section{Sutura de tejido celular subcutáneo y piel}

En lo que respecta a la sutura del tejido celular subcutáneo, se considera que debe ser suturado si el espesor del mismo es mayor de $2 \mathrm{~cm}$. Se ha observado que el cierre del subcutáneo mediante puntos separados es superior a la sutura continua, en lo que respecta a las 


\section{TÉCNICAS QUIRÚRGICAS EN CESÁREA SEGMENTARIA. REVISIÓN DE EVIDENCIAS}

Cuadro 5. Base de datos Cochrane de revisiones sistemáticas

Técnica quirúrgica en cesárea. Cierre del peritoneo

\begin{tabular}{|c|c|c|c|c|}
\hline Año & Autores & Tipo y n de ensayos & $\mathrm{N}$ casos & Parámetros y resultados \\
\hline \multirow{14}{*}{2014} & \multirow{14}{*}{$\begin{array}{l}\text { Bamigboye } \\
\text { y cols.(37) }\end{array}$} & & & No cierre de ambos peritoneos vs cierre de ambos peritoneos \\
\hline & & $\mathrm{A} / \mathrm{C} 4$ & 282 & $\begin{array}{c}\text { Adherencias posoperatorias vs no adherencias } \\
\text { RR: 0,99 (IC 0,76-1,29 95\%) NS }\end{array}$ \\
\hline & & $\mathrm{A} / \mathrm{C} 13$ & 14.906 & $\begin{array}{l}\boldsymbol{\nabla} \text { Tiempo operatorio. RR }=\text { Pr.-5,81 min (IC }-7,68 \text { a }-3,93 \min 95 \% \text { ) } \\
\nabla \text { Estancia hospitalaria Pr. }-0,26 \text { días (IC }-0,47 \text { a 0,05 días } 95 \% \text { ) }\end{array}$ \\
\hline & & $\mathrm{A} / \mathrm{C} \quad 1$ & 112 & $\nabla$ Dolor pélvico crónico RR=0,49 (IC 0,25-0,98 95 \%) \\
\hline & & & & No cierre de peritoneo visceral solo vs cierre de ambos peritoneos \\
\hline & & $\mathrm{A} / \mathrm{C} 2$ & 157 & $\Delta$ Formación de adherencias RR=2,49 (IC 1,49-4,16 95\%) \\
\hline & & $\mathrm{A} / \mathrm{C} \quad 1$ & 544 & $\begin{array}{l}\Delta \text { Tiempo operatorio } \mathrm{RR}=-6,30 \mathrm{~min}(\mathrm{IC}-9,22 \text { a }-3,38 \min 95 \%) \\
\boldsymbol{\nabla} \text { Estancia hospitalaria } \mathrm{RR}=-0,70 \text { días (IC }-0,98 \text { a }-0,42 \text { 95 \%) }\end{array}$ \\
\hline & & $\mathrm{A} / \mathrm{C} 2$ & & $\boldsymbol{\nabla}$ Infección herida operatoria $\mathrm{RR}=0,36$ (IC 0,14- 0,89 95\%) \\
\hline & & $\mathrm{A} / \mathrm{C} \quad 3$ & 889 & $\boldsymbol{\nabla}$ NS Fiebre postoperatoria RR=0,60 (IC 0,29-1,27 95\%) \\
\hline & & & & No cierre de peritoneo parietal solo vs cierre de ambos peritoneos \\
\hline & & $\mathrm{A} / \mathrm{C}$ & 248 & $\boldsymbol{\nabla}$ Tiempo operatorio $\mathrm{RR}=$ Prom. $-5,10 \mathrm{~min}(\mathrm{IC}-8,71 \mathrm{a}-1,4995 \%)$ \\
\hline & & $\mathrm{A} / \mathrm{C}$ & 325 & $\boldsymbol{\nabla}$ Dolor postoperatorio $\mathrm{RR}=0.45$ (IC 0,31- 0,66 $95 \%$ ) \\
\hline & & & & $\begin{array}{l}\text { No cierre del peritoneo visceral cuando } \\
\text { se cierra el peritoneo parietal }\end{array}$ \\
\hline & & $\mathrm{A} / \mathrm{C} \quad 1$ & 297 & $\begin{array}{l}\boldsymbol{\nabla} \text { Frecuencia miccional } \mathrm{RR}=0,24 \text { (IC } 0,13-0,4595 \%) \\
\boldsymbol{\nabla} \text { Urgencia miccional } \mathrm{RR}=0,30 \text { (IC } 0,18-0,5195 \%) \\
\nabla \text { Incontinencia urinaria } \mathrm{RR}=0,45(\mathrm{IC} 0,21-0,9695 \%)\end{array}$ \\
\hline
\end{tabular}

$\mathrm{A} / \mathrm{C}=$ Ensayo aleatorizado y controlado; $\mathrm{RR}=$ Riesgo relativo; $\mathrm{IC}=$ Intervalo de confianza; $\mathrm{NS}=$ No significativo;

$\boldsymbol{\nabla}=$ Disminución; $\quad \boldsymbol{\Delta}=$ Aumento.

complicaciones posteriores (44). En una revisión de Cochrane de 2012, no hubo ninguna diferencia en el uso de grapas no absorbibles versus sutura intradérmica absobible de la piel, en lo relativo a infección de la herida, dolor y aspecto estético, pero si se observó una mayor incidencia de separación de la herida operatoria y por consiguiente de resutura con el uso de grapas, si son removidas antes del cuarto día posoperatorio. Se observó una mayor incidencia de cicatriz hipertrófica o queloide en la sutura de piel con puntos separados en comparación con la sutura intradérmica. No hubo suficientes evidencias para comparar otros tipos de suturas y materiales utilizados, ni tampoco suficientes evidencias para concluir cuál es el mejor método o el tipo de sutura para el cierre de la herida operatoria en la cesárea $(45,46)$ (Cuadro 6). 
Cuadro 5. Base de datos Cochrane de revisiones sistemáticas

Técnica quirúrgica en cesárea. Cierre del peritoneo

\begin{tabular}{|c|c|c|c|c|}
\hline Año & Autores & Tipo y n de ensayos & $\mathrm{N}$ casos & Parámetros y resultados \\
\hline \multirow{7}{*}{2012} & \multirow{7}{*}{$\begin{array}{l}\text { Mackeen y } \\
\text { cols. (45) }\end{array}$} & \multirow{5}{*}{ A 6} & \multirow{5}{*}{916} & Técnicas de sutura de piel y materiales utilizados \\
\hline & & & & Grapas no absorbibles vs sutura absorbible \\
\hline & & & & Infección RR: 0,85 (IC 0,43-1,71 95 \%) NS \\
\hline & & & & A Dehiscencia RR: 3,82 (IC 2,05-7,12 95 \%) \\
\hline & & & & $\Delta$ Resutura RR: 4,98 (IC 1,82-13,61 95\%) \\
\hline & & \multirow{2}{*}{ A 1} & \multirow{2}{*}{62} & Sutura a puntos separados vs intradérmica \\
\hline & & & & Cicatriz hipertrófica: RR: 1,85 (1,33-2,58 IC $95 \%)$ \\
\hline
\end{tabular}

$\mathrm{A}=$ Aleatorizado; $\mathrm{A} / \mathrm{C}=$ Aleatorizado y controlado; $\mathrm{RR}=$ Riesgo relativo; $\mathrm{IC}=$ Intervalo de confianza al $95 \%$; Prom. $=$ Promedio;

NS= No significativo; $\quad \boldsymbol{\nabla}=$ Disminución $\boldsymbol{\Delta}=$ Aumento

\section{CONCLUSIONES}

De la revisión sobre las distintas técnicas quirúrgicas realizadas en los últimos 10 años, con especial referencia a la técnica simplificada Misgav-Ladach comparada con la técnica de Pfannenstiel-Kerr, se puede llegar a las siguientes conclusiones, basadas en anteriores y nuevas evidencias:

1. La cesárea se debe realizar utilizando preferiblemente una incisión transversa cutánea ya que se asocia a menor riesgo de hernia incisional y mejor efecto cosmético comparado con la incisión media. La incisión cutánea de elección en la técnica Misgav-Ladach es la de Joel Cohen.

2. Los planos del tejido celular subcutáneo y aponeurosis se deben incidir primero en la línea media con bisturí y luego la apertura debe ser preferiblemente completada con separación digital de la grasa y de la fascia del recto anterior, maniobras que se acompañan con una menor lesión de vasos sanguíneos y un menor dolor posoperatorio agudo, crónico y neuropático.

3. La separación de los músculos rectos debe hacerse, con preferencia, digitalmente y traccionando lateralmente, sobre todo en pacientes sometidas a su primera cesárea.

4. El peritoneo parietal se trata de abrir digitalmente o con la ayuda de una pinza de Crile, empezando en la parte más alta del mismo, evitando el corte con tijera o electro bisturí. Se supone que con esta técnica hay menor riesgo de lesión intestinal y de vejiga.

5. No es recomendable disecar el peritoneo visceral, la plica o fondo vesical, la parte inferior de los rectos abdominales ni desinsertar los músculos piramidales.

6. La extensión de la incisión uterina a nivel del segmento debe realizarse, con preferencia, digitalmente, ya que reduce la pérdida sanguínea, la incidencia de hemorragia poscesárea y la necesidad de transfusión.

7. El alumbramiento debe ser dirigido, no manual, para reducir el riesgo de endometritis.

8. No se recomienda la exteriorización del útero para la histerorrafia, ya que se asocia con mayor dolor posoperatorio. 


\section{TÉCNICAS QUIRÚRGICAS EN CESÁREA SEGMENTARIA. REVISIÓN DE EVIDENCIAS}

9. La efectividad y seguridad del cierre en un plano de la histerorrafia no está aclarada, por lo tanto, se sugiere reparación en 2 planos continuos.

10. No se recomienda la sutura rutinaria del peritoneo visceral y parietal, ya que esto reduce el tiempo operatorio, la necesidad de mayor analgesia y no se ha visto una mayor frecuencia de adherencias intraabdominales.

11. A menos que se trate de una incisión media infraumbilical, se recomienda no aproximar ni suturar el músculo recto abdominal, a fin de evitar dolor posoperatorio.

12. La aponeurosis se sigue cerrando con sutura continua no cruzada, preferiblemente con poliglactina o material no absorbible, con cierta separación entre los puntos.

13. No se recomienda el cierre rutinario del tejido celular subcutáneo, a menos que su grosor sea mayor de $2 \mathrm{~cm}$., en cuyo caso debe hacerse a puntos separados.

14. No existe una técnica ideal para el cierre de la piel.

\section{Agradecimiento}

El autor expresa su agradecimiento a la Dra. Ofelia Uzcátegui por la revisión del manuscrito.

\section{REFERENCIAS}

1. Ventura W. Estudio comparativo entre cesárea tipo Misgav-Ladach y cesárea tipo Kerr. An Fac Med [Internet]. 2009 [consultado febrero de 2021]; 70(3); 199-204. Disponible en: http://www.scielo. org.pe/scielo.php?script $=$ sci_arttext\&pid $=\mathrm{S} 1025$ 55832009000300008\&lng=es.

2. Kerr JMM. The technique of cesarean section, with special reference to the lower uterine segment. Am J Obstet Gynecol 1926; 12: 729-734.
3. Stark M. Technique of Caesarean section: the Misgav Ladach method. In: Popkin DR, Peddle LJ, eds. Women's health today: perspectives on current research and clinical practice. Proc. XIVWorld Congress Gynecol Obstet: Montreal. New York and London: Parthenon, 1994: 81-85.

4. Holmgren G, Sjöholm L, Stark M. The Misgav Ladach method for cesarean section: method description. Acta Obstet Gynecol Scand. 1999; 78(7):615-621. https://doi. org/10.1177/004947559602600404

5. Joel-Cohen S. Abdominal and vaginal hysterectomy. New techniques based on time and motion studies. William Heinemann Medical Books, 1972; 170.

6. Suarez D. Cesárea. En: Zighelboim I, Guariglia D, editores. Clínica Obstétrica 2da ed. Caracas: Editorial Disinlimed CA, 2005 p.583-589.

7. Nieto T, Cañete ML, Valero FJ, Melchor JC. Cesárea. En: Bajo JM, Melchor JC, Mercé LT, editores. Fundamentos de Obstetricia (SEGO). $1^{\text {a }}$ Ed. Madrid: Editorial Gráficas Marte SRL, 2007, p. 793-807.

8. Yapca OE, Topdagi YE, Al RA. Fetus delivery time in extraperitoneal versus transperitoneal cesarean section: a randomized trial. J Matern Fetal Neonatal Med. 2020; 33(4):657-663. doi: 10.1080/14767058.2018.1499718.

9. Delplanque S, Le Lous M, Isly H, Coiffic J, Lassel L, Levêque J, et al. Operative Technique and Experience of One Referral Center With Vaginal Cesarean Delivery. Obstet Gynecol. 2020; 135(2):409-414. doi: 10.1097/ AOG.0000000000003650.

10. Naki MM, Api O, Celik H, Kars B, Yaşar E, Unal O. Comparative study of Misgav-Ladach and PfannenstielKerr cesarean techniques: a randomized controlled trial. J Matern Fetal Neonatal Med. 2011; 24(2):239-244. doi: 10.3109/14767058.2010.482612.

11. Hudić I, Bujold E, Fatušić Z, Skokić F, Latifagić A, Kapidžić M, et al. The Misgav-Ladach method of cesarean section: a step forward in operative technique in obstetrics. Arch Gynecol Obstet. 2012; 286(5):11411146. doi: 10.1007/s00404-012-2448-6.

12. CORONIS Collaborative Group, Abalos E, Addo V, Brocklehurst P, El Sheikh M, Farrell B, Gray S, et al. Caesarean section surgical techniques (CORONIS): a fractional, factorial, unmasked, randomised controlled trial. Lancet. 2013; 382(9888):234-248. doi: 10.1016/ S0140-6736(13)60441-9. 
13. CORONIS collaborative group, Abalos E, Addo V, Brocklehurst P, El Sheikh M, Farrell B, Gray S, et al. Caesarean section surgical techniques: 3 year follow-up of the CORONIS fractional, factorial, unmasked, randomised controlled trial. Lancet. 2016; 388(10039):62-72. doi: 10.1016/S01406736(16)00204-X.

14. Tuuli MG, Liu J, Stout MJ, Martin S, Cahill AG, Odibo AO, et al. Randomized Trial Comparing Skin Antiseptic Agents at Cesarean Delivery. N Engl J Med. 2016; 374(7):647-655. doi: 10.1056/NEJMoa1511048.

15. Hadiati DR, Hakimi M, Nurdiati DS, Masuzawa Y, da Silva Lopes K, Ota E. Skin preparation for preventing infection following caesarean section. Cochrane Database Syst Rev. 2020; 6(6):CD007462. doi: 10.1002/14651858.CD007462.pub5.

16. Maaløe N, Aabakke AJ, Secher NJ. Midline versus transverse incision for cesarean delivery in low-income countries. Int J Gynaecol Obstet. 2014; 125(1):1-2. doi: 10.1016/j.ijgo.2013.09.030.

17. Mathai M, Hofmeyr GJ, Mathai NE. Abdominal surgical incisions for caesarean section. Cochrane Database Syst Rev. 2013; (5):CD004453. doi: 10.1002/14651858. CD004453.pub3.

18. Gizzo S, Andrisani A, Noventa M, Di Gangi S, Quaranta $\mathrm{M}$, Cosmi E, et al. Caesarean section: could different transverse abdominal incision techniques influence postpartum pain and subsequent quality of life? A systematic review. PLoS One. 2015; 10(2):e0114190. doi: 10.1371/journal.pone.0114190.

19. Belci D, Di Renzo GC, Stark M, Đurić J, Zoričić D, Belci M, et al. Morbidity and chronic pain following different techniques of caesarean section: A comparative study. J Obstet Gynaecol. 2015; 35(5):442-446. doi: 10.3109/01443615.2014.968114.

20. AbdElaal NK, Ellakwa HE, Elhalaby AF, Shaheen AE, Aish AH. Scalpel versus diathermy skin incision in Caesarean section. J Obstet Gynaecol. 2019; 39(3):340344. doi: 10.1080/01443615.2018.1527298.

21. Hajibandeh S, Hajibandeh S, Maw A. Diathermy versus scalpel for skin incision in patients undergoing open inguinal hernia repair: A systematic review and metaanalysis. Int J Surg. 2020; 75:35-43. doi: 10.1016/j. ijsu.2020.01.020.
22. Yazici Yilmaz F, Aydogan Mathyk B, Yildiz S, Yenigul NN, Saglam C. Postoperative pain and neuropathy after caesarean operation featuring blunt or sharp opening of the fascia: a randomised, parallel group, double-blind study. J Obstet Gynaecol. 2018; 38(7):933-939. doi: 10.1080/01443615.2018.1437125.

23. Berghella V. Cesarean birth. Surgical technique. En: Berghella V, Lockwood Ch, Barrs V, section editors. Obstetrics. Waltham, Massachusetts: UpToDate; 2016. Disponible en: https://www.uptodate.com/contents/ cesarean-birth-surgical-technique

24. Mahajan NN. Justifying formation of bladder flap at cesarean section? Arch Gynecol Obstet. 2009; 279(6):853-855. doi: 10.1007/s00404-008-0838-6.

25. Kadir RA, Khan A, Wilcock F, Chapman L. Is inferior dissection of the rectus sheath necessary during Pfannenstiel incision for lower segment Caesarean section? A randomised controlled trial. Eur J Obstet Gynecol Reprod Biol. 2006; 128(1-2):262-266. doi: 10.1016/j.ejogrb.2006.02.018.

26. Chicaud B, Roux C, Rudigoz RC, Huissoud C. [Blunt or sharp expansion of cesarean section: a comparative study]. J Gynecol Obstet Biol Reprod (Paris). 2013; 42(4):366-371. French. doi: 10.1016/j.jgyn.2013.03.006.

27. Asıcıoglu O, Gungorduk K, Asıcıoglu BB, Yildırım G, Gungorduk OC, Ark C. Unintended extension of the lower segment uterine incision at cesarean delivery: a randomized comparison of sharp versus blunt techniques. Am J Perinatol. 2014; 31(10):837-844. doi: 10.1055/s-0033-1361934.

28. Dodd JM, Anderson ER, Gates S, Grivell RM. Surgical techniques for uterine incision and uterine closure at the time of caesarean section. Cochrane Database Syst Rev. 2014; (7):CD004732. doi: 10.1002/14651858. CD004732.pub3.

29. Ezegwui HU, Ogbuefi FC. Routine cervical dilatation during elective caesarean section. Should we continue? J Obstet Gynaecol. 2015; 35(2):150-152. doi: 10.3109/01443615.2014.937333.

30. Di Spiezio Sardo A, Saccone G, McCurdy R, Bujold E, Bifulco G, Berghella V. Risk of Cesarean scar defect following single- vs double-layer uterine closure: systematic review and meta-analysis of randomized controlled trials. Ultrasound Obstet Gynecol. 2017; 50(5):578-583. doi: 10.1002/uog. 17401. 


\section{TÉCNICAS QUIRÚRGICAS EN CESÁREA SEGMENTARIA. REVISIÓN DE EVIDENCIAS}

31. Vachon-Marceau C, Demers S, Bujold E, Roberge S, Gauthier RJ, Pasquier JC, et al. Single versus doublelayer uterine closure at cesarean: impact on lower uterine segment thickness at next pregnancy. Am J Obstet Gynecol. 2017; 217(1):65.e1-65.e5. doi: 10.1016/j. ajog.2017.02.042.

32. Walsh CA, Walsh SR. Extraabdominal vs intraabdominal uterine repair at cesarean delivery: a metaanalysis. Am J Obstet Gynecol. 2009; 200(6):625.e1-8. doi: 10.1016/j. ajog.2009.01.009.

33. Coutinho IC, Ramos de Amorim MM, Katz L, Bandeira de Ferraz AA. Uterine exteriorization compared with in situ repair at cesarean delivery: a randomized controlled trial. Obstet Gynecol. 2008; 111(3):639-647. doi: 10.1097/AOG.0b013e31816521e2.

34. Terán J, Turmero J. Cesárea segmentaria sin cierre de ambos peritoneos. Rev Obstet Ginecol Venez. 1998; 58(1):13-15.

35. Pagés G, Aller J, Jiménez R, Rasines MI, Martell A, Aller B. Cirugías abdominales obstétrico-ginecológicas sin cierre de peritoneos, Rev Obstet Ginecol Venez. 2001; 61(1): 31-34.

36. Malek-Mellouli M, Ibrahima S, Ben Amara F, Néji K, Bouchneck M, Youssef A, et al. Vers une simplification de la technique de césarienne : suture péritonéale ou non ? [Towards a simplification of caesarean section technique: non-closure of peritoneum?]. J Gynecol Obstet Biol Reprod (Paris). 2011; 40(6):541-548. French. doi: 10.1016/j.jgyn.2011.06.004.

37. Bamigboye AA, Hofmeyr GJ. Closure versus nonclosure of the peritoneum at caesarean section: shortand long-term outcomes. Cochrane Database Syst Rev. 2014; (8):CD000163. doi: 10.1002/14651858. CD000163.pub2.

38. Kapustian V, Anteby EY, Gdalevich M, Shenhav S, Lavie O, Gemer O. Effect of closure versus nonclosure of peritoneum at cesarean section on adhesions: a prospective randomized study. Am J Obstet Gynecol. 2012; 206(1):56.e1-4. doi: 10.1016/j.ajog.2011.07.032.

39. Kurek Eken M, Özkaya E, Tarhan T, İçöz Ş, Eroğlu Ş, Kahraman ŞT, et al. Effects of closure versus nonclosure of the visceral and parietal peritoneum at cesarean section: does it have any effect on postoperative vital signs? A prospective randomized study. J Matern Fetal Neonatal Med. 2017; 30(8):922-926. doi: 10.1080/14767058.2016.1190826.
40. Lyell DJ, Caughey AB, Hu E, Daniels K. Peritoneal closure at primary cesarean delivery and adhesions. Obstet Gynecol. 2005; 106(2):275-280. doi: 10.1097/01. AOG.0000171120.81732.4c.

41. Tulandi T, Hum HS, Gelfand MM. Closure of laparotomy incisions with or without peritoneal suturing and secondlook laparoscopy. Am J Obstet Gynecol. 1988; 158(3 Pt 1):536-537. doi: 10.1016/0002-9378(88)90020-8.

42. Omran EF, Meshaal H, Hassan SM, Dieb AS, Nabil H, Saad H. The effect of rectus muscle reapproximation at cesarean delivery on pain perceived after operation: a randomized control trial. J Matern Fetal Neonatal Med. 2019; 32(19):3238-3243. doi: 10.1080/14767058.2018.1461829.

43. Hodgson NC, Malthaner RA, Ostbye T. The search for an ideal method of abdominal fascial closure: a meta-analysis. Ann Surg. 2000; 231(3):436-442. doi: 10.1097/00000658-200003000-00018.

44. Alalfy M, Elgazzar A, Fares T, Nagy O, Ellithy A, Lasheen Y, et al. Effect of subcutaneous tissue closure technique in cesarean section on postoperative wound complications in obese Egyptian women. J Matern Fetal Neonatal Med. 2019; 32(15):2452-2459. doi: 10.1080/14767058.2018.1438399.

45. Mackeen AD, Berghella V, Larsen ML. Techniques and materials for skin closure in caesarean section. Cochrane Database Syst Rev. 2012 Nov 14;11(11):CD003577. doi: 10.1002/14651858.CD003577.pub3.

46. Cooper SM, Blanchard CT, Szychowski JM, Tita ATN. Does Time of Wound Complication after Cesarean Delivery Differ by Type of Skin Closure? Am J Perinatol. 2019; 36(9):981-984. doi: 10.1055/s-0038-1675767
Recibido 29 de abril 2021

Aprobado 20 de junio de 2021 